IAA disrupts mitochondrial oxidative phosphorylation program via the Akt/mTOR axis, thereby suppressing the differentiation of ISC and organoid formation (IDDF2021-ABS-0074 Figure 6, IDDF2021-ABS-0074 Figure 7). Activation of Akt reverses IAA-induced impairment of mitochondrial energetics, stem cell maintenance and epithelial renewal in chronically-stressed mice (IDDF2021-ABS-0074 Figure 8).

Conclusions Our results connect gut dysbiosis and IAA to ISC dysfunction during psychological stress and suggest that modulating gut microbial metabolism may prevent the gut from the deleterious effect of stressful events.

\section{IDDF2021-ABS-0076 LNCRNA TNFRSF10A-AS1 MEDIATES TUMORIGENESIS OF GASTRIC CANCER BY DIRECTLY BINDING TO MPZL1 AND ASSOCIATES WITH PATIENT OUTCOMES}

${ }^{1}$ Donglei Sun*, ${ }^{2}$ Hongyan Gou, ${ }^{1}$ Dandan Wang, ${ }^{3} J u n$ Yu. ${ }^{1}$ The Second Hospital of Hebei Medical University, China; ${ }^{2}$ CUHK Shenzhen Research Institute, The Chinese University of Hong Kong, China; ${ }^{3}$ The Chinese University of Hong Kong, Hong Kong, China

\subsection{6/gutjn--2021-IDDF.32}

Background LncRNA was known to be closely associated with the progression of human tumors. The role of new IncRNA TNFRSF10A-AS1 in the pathogenesis and progression of gastric tumor is still unclear. The aim of this study was to investigate the function of TNFRSF10A-AS1 and the underlying mechanism in the pathogenesis and progression of gastric cancer.

Methods The clinical impact of TNFRSF10A-AS1 was assessed in 105 patients with gastric cancer. The biological function of TNFRSF10A-AS1 was studied in vitro and in vivo. TNFRSF10A-AS1 downstream effector were identified by RNA FISH, RNA sequencing, RNA pulldown and rescue assay.

Results TNFRSF10A-AS1was upregulated in gastric cancer cell lines and tissues. Multivariate analysis showed that gastric cancer patients with TNFRSF10A-AS1 overexpression had a significantly shortened survival. TNFRSF10A-AS1 significantly promoted gastric cancer cell proliferation, cell-cycle transformation, and migration/invasion, but suppressed cell apoptosis. Silencing TNFRSF10A-AS1 expression exerted opposite effects in vitro and significantly inhibited xenograft tumor growth in nude mice. Mechanically, TNFRSF10A-AS1 directly bound to MPZL1 and activated MPZL1 transcription. Knockdown MPZL1 abrogated the effect of TNFRSF10A-AS1 in the tumor-promoting function.

Conclusions TNFRSF10A-AS1 directly binds to oncogenic MPZL1 to induce its expression. TNFRSF10A-AS1 plays a pivotal oncogenic role in gastric carcinogenesis and is an independent prognostic factor for gastric cancer patients.

\section{IDDF2021-ABS-0094 VIRULENCE FACTORS AND RESISTANCE GENES CARRIED DIFFERENCES OF KLEBSIELLA PNEUMONIAE ISOLATED FROM PATIENTS WITH PNEUMONIA AND PYOGENIC LIVER ABSCESS}

Lin Gan*, Chao Yan, Jinghua Cui, Guanhua Xue, Junxia Feng, Hanqing Zhao, Yanling Feng, Jing Yuan. Capital Institute of Pediatrics, China

10.1136/gutjnl-2021-IDDF.33
Background The bacterium Klebsiella pneumoniae of family Enterobacteriacea is a well-known opportunistic pathogen that colonizes intestinal and respiratory tract. While K. pneumoniae is a common cause of nosocomial and community-acquired infections, including diarrhea, pneumonia and pyogenic liver abscess, little is known about the population structure of this bacterium. Thus it is likely that the epidemiological characteristics of $\mathrm{K}$. pneumoniae isolate from carriers and clinic patients, when combined to their genomic information, might provide some insight into pathogenic Klebsiella prevention and control.

Methods Two hundred and thirty-two K. pneumoniae isolates (including 38 isolates from carriers, 124 isolates from pyogenic liver abscess patients and 70 isolates from pneumonia patients) were collected from 9 provinces of China in 20132020. Sequencing was performed on the Illumina Hiseq PE150 platform, and the genome sequences were assembled by SOAP denovo. Multilocus sequence typing (MLST) analysis was done by submitting sequences to the Institute Pasteur K. pneumoniae MLST database. Pan-genome analysis was performed by software Snippy, Gubbins and Roary, and the gene contents were identified by software VFanalyzer, Resfinder and PlasmidFinder.

Results The 232 isolates were subtyped into 74 STs. The isolates from different sources have their own STs, and the predominant subtypes of liver abscess patients and pneumonia patients were ST23 and ST11, respectively. PCA analysis ( $p$ $=0.001)$ on accessory gene content also distinguished the three phylogroups, which are consistent with the source of isolates. The isolates collected from liver abscess patients carried significantly more $(p=0.000)$ virulence factors, and the isolates sourced from pneumonia patients harbored significantly more $(p=0.000)$ resistance genes and replicons. Besides, there was a strong link between Salmonchelin and the isolates sourced from liver abscess patients. Ninety-eight percent isolates of liver abscess strongly correlated STs and only two percent isolates of pneumoniae correlated STs carried Salmonchelin.

Conclusions These data provide genomic support for the proposal that isolates collected from carrier, liver abscess and pneumoniae patients have their distinct genomic features. And the isolates from different sources are largely nonoverlapping.

\section{IDDF2021-ABS-0097 P38 $\alpha$ DEFICIENCY IN MACROPHAGES AMELIORATES MURINE EXPERIMENTAL COLITIS BY REGULATING INFLAMMATION AND IMMUNE PROCESS}

Wei Chen*, Jinshui Zhu, Jing Zhang, Rui Liang, Youcai Yi, Xiaoyu Chen, Huining Fan. Shanghai Jiao Tong University Affiliated Sixth People's Hospital, China

\subsection{6/gutjnl-2021-IDDF.34}

Background $\mathrm{P} 38 \alpha$ is a mitogen-activated protein kinase (MAPK) that mediates inflammatory responses. P38 $\alpha$ alterations have been associated with inflammation-related diseases. However, the role of macrophages-derived p38 $\alpha$ in dextran sulfate sodium (DSS)-induced murine experimental colitis remains unclear. We characterized the role of macrophagesderived $\mathrm{p} 38 \alpha$ in DSS-induced colitis.

Methods The expression of macrophage-derived $\mathrm{p} 38 \alpha$ in human colitis and normal tissues was measured by 
immunohistochemistry (IHC) and fluorescence in situ hybridization (FISH) analysis. Macrophage-specific p38 $\alpha$ knockout $\left(\mathrm{p} 38 \alpha^{\Delta \mathrm{M \phi}}\right.$ ) and wild type (WT) mice administrated by $3 \%$ DSS were used to establish experimental colitis. The alterations in inflammatory cytokines, intestinal epithelial barrier, cell proliferation and cell apoptosis between $\mathrm{p} 38 \alpha^{\Delta \mathrm{M} \phi}$ and WT groups were determined by IHC, immunofluorescence (IF), TdT-mediated dUTP Nick-End Labeling (TUNEL) and Western blot analyses. The enriched pathways between p $38 \alpha^{\Delta \mathrm{M} \phi}$ and WT groups were identified by RNA-seq and KEGG analysis.

Results p38 $\alpha$ was co-localized with CD68 in the cytoplasm and their co-expression indicated an increased level in colitis tissues as compared with the normal tissues (IDDF2021-ABS0097 Figure 1. Macrophage $\mathrm{p} 38 \boldsymbol{\alpha}$ is upregulated in human colitis colon tissues. (A) Immunohistochemistry images of pp38 $\alpha$. (B) p-p38 $\alpha$ protein expression in colitis $(n=10)$ and normal colon tissues $(n=5)$. (C) FISH images of CD68 and p38 $\alpha$. (D) Analysis of the association between CD68 and p38 $\alpha$. (E) The expression of CD68, p38 $\alpha$ and CD68-p38 $\alpha$ in colitis $(n=10)$ and normal colon tissues $(n=5)$. $* P<$ $0.05)$. P38 deficiency in macrophages was sufficient to suppress the exacerbated clinical symptoms and inflammation responses in experimental colitis (IDDF2021-ABS-0097 Figure
2. Macrophage p $38 \alpha$ protects against DSS-induced colitis. (A) Body weight changes. (B) Disease active index. (C) Gross morphology images of colons. (D) Colon lengths. (E) H\&E staining. (F) Histology scores. ${ }^{*} P<0.05$ and $* P<0.01$ ), followed by reducing cytokine release (IDDF2021-ABS-0097 Figure 3. Macrophage p38 $\alpha$ deletion inhibits pro-inflammatory cytokine production in DSS induced colitis. (A-C) The expression levels of TNF - $\boldsymbol{\alpha}$, IL-6 and IL-1 $\beta$. ${ }^{*} P<0.05$, $* * P<0.01, * * P<0.001$.), increasing MUC-2 and Claudin-2 secretion (IDDF2021-ABS-0097 Figure 4. Macrophage p38 $\alpha$ deficiency induces the secretion of muc-2 and claudin- 2 . The mRNA expression (A) and IHC images (B) of muc-2 and claudin-2. (C) Western blot of claudin-2 protein levels. (D) IF images of muc-2 and claudin-2. $* P<0.05$ and $* * P<$ 0.01.) and promoting colonic mucosa repair (IDDF2021-ABS0097 Figure 5. Macrophage p38 $\alpha$ deficiency affects the proliferation and apoptosis of IECs. (A) Ki-67 expression. (B) The apoptosis of IECs. $* P<0.05$ and $* * * P<0.001)$. Further investigations validated that the immune processrelated factors were upregulated in colon tissues from $\mathrm{p} 38 \alpha^{\Delta \mathrm{M} \phi}$ group as compared with the WT group (IDDF2021-ABS-0097 Figure 6. Macrophage p38 $\alpha$ deficiency enhances immune system process during colitis. (A) Heatmap. (B) Volcano plotting. (C,D) Go analysis), (IDDF2021-ABS-

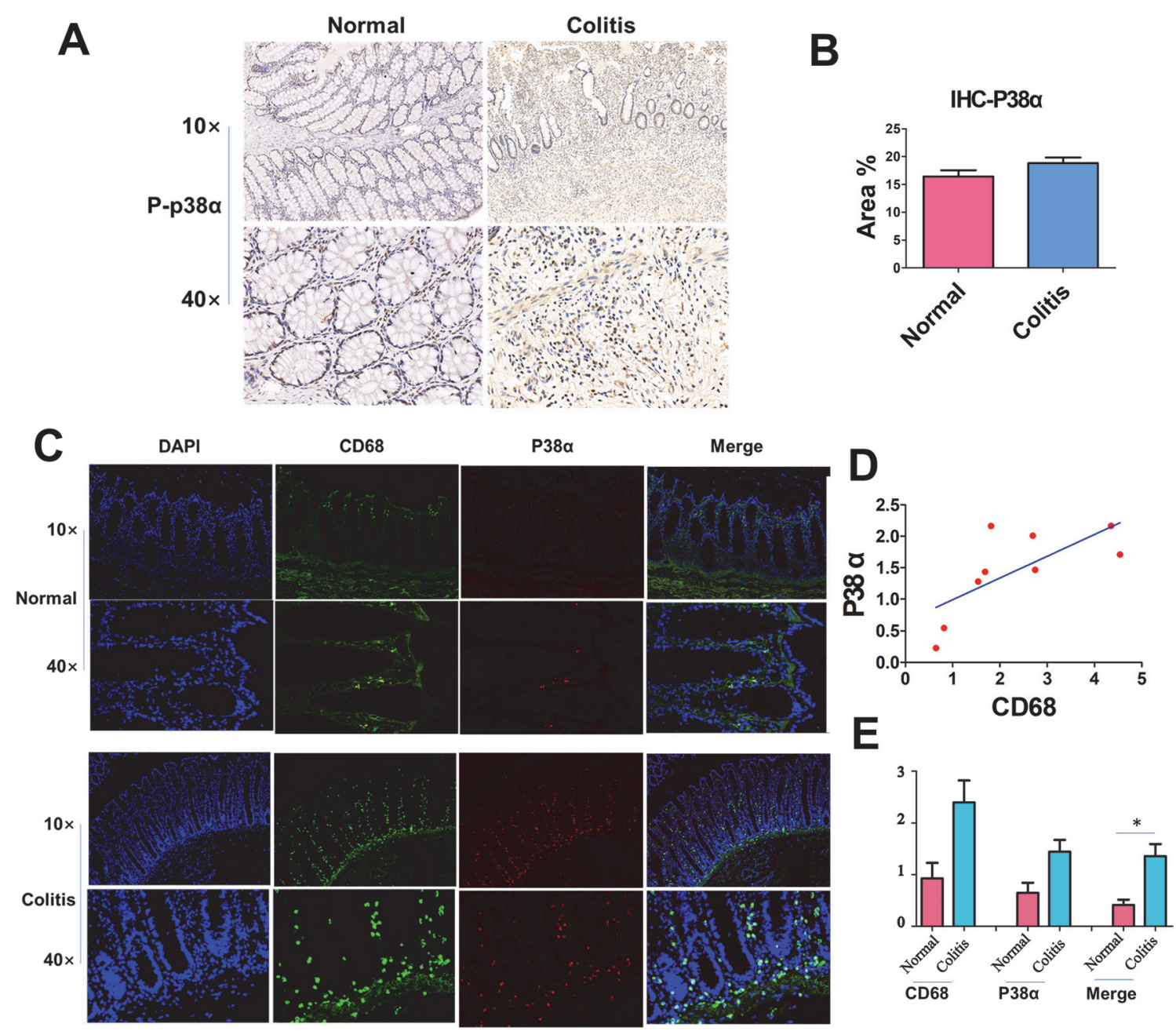

Abstract IDDF2021-ABS-0097 Figure 1 

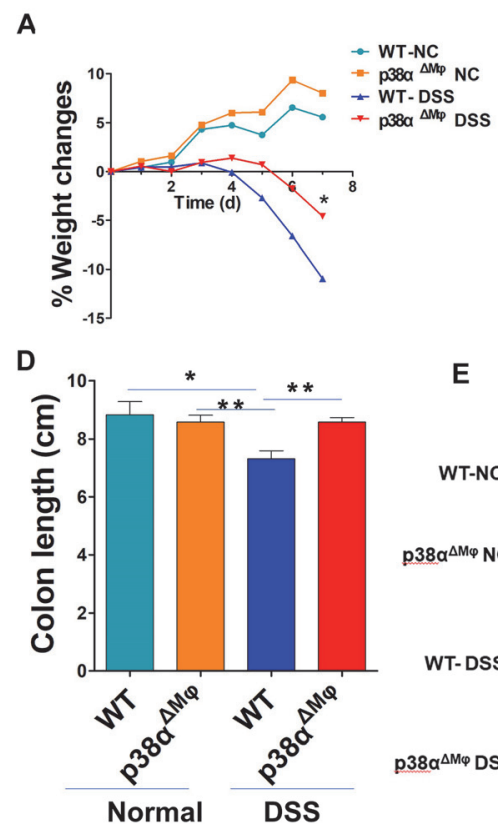

E
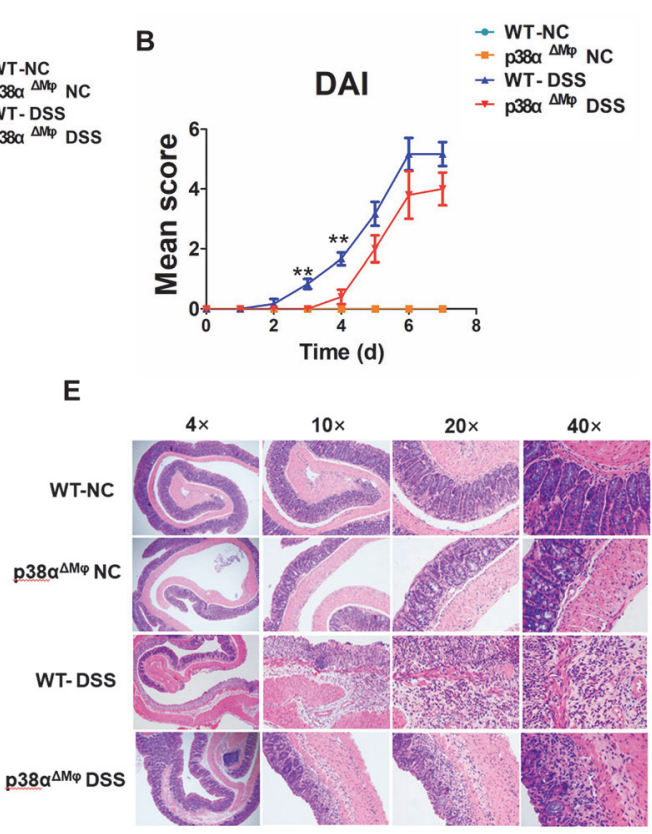
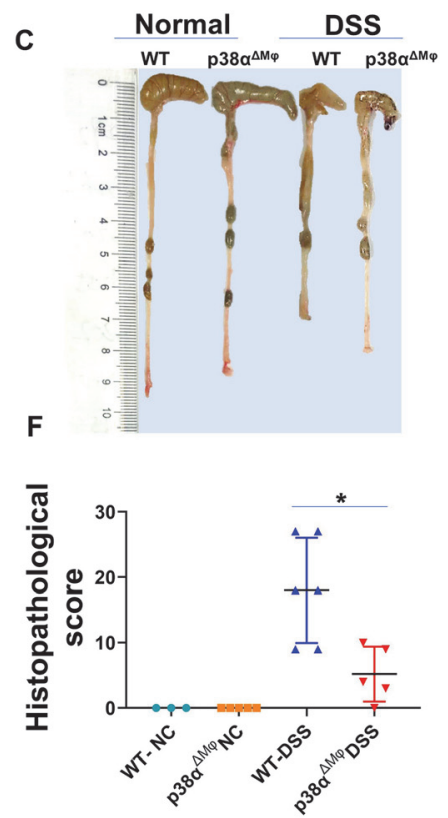

Abstract IDDF2021-ABS-0097 Figure 2
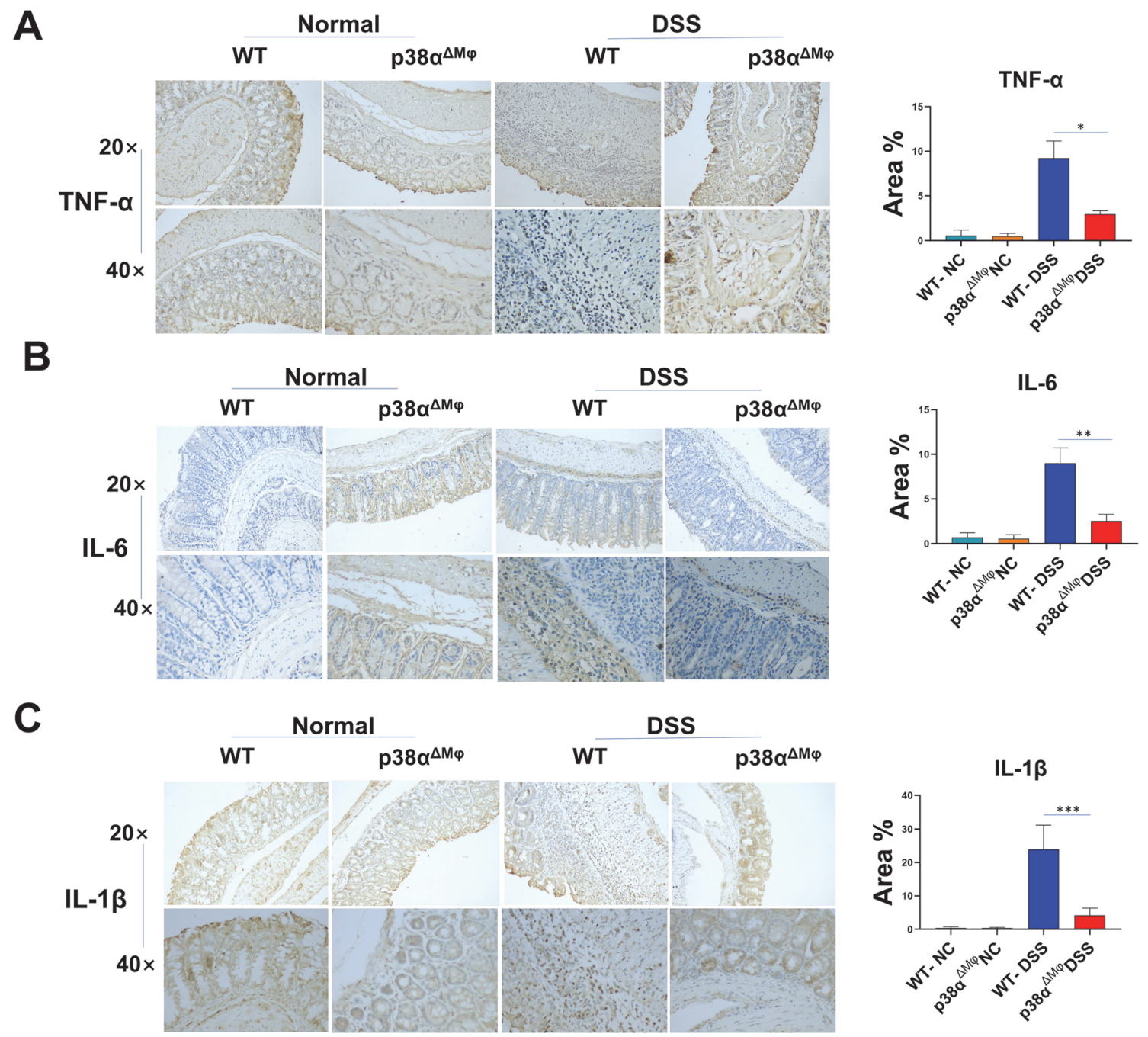

Abstract IDDF2021-ABS-0097 Figure 3 
A
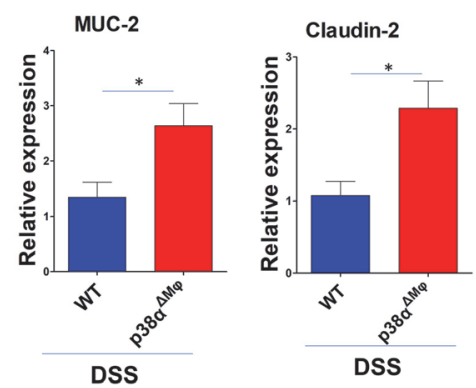

Claudin-2

$40 \times$

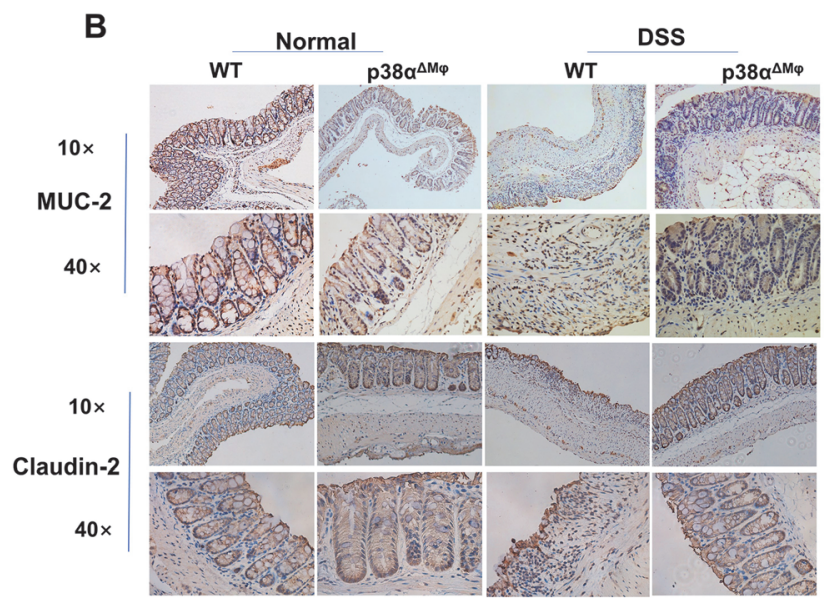

D
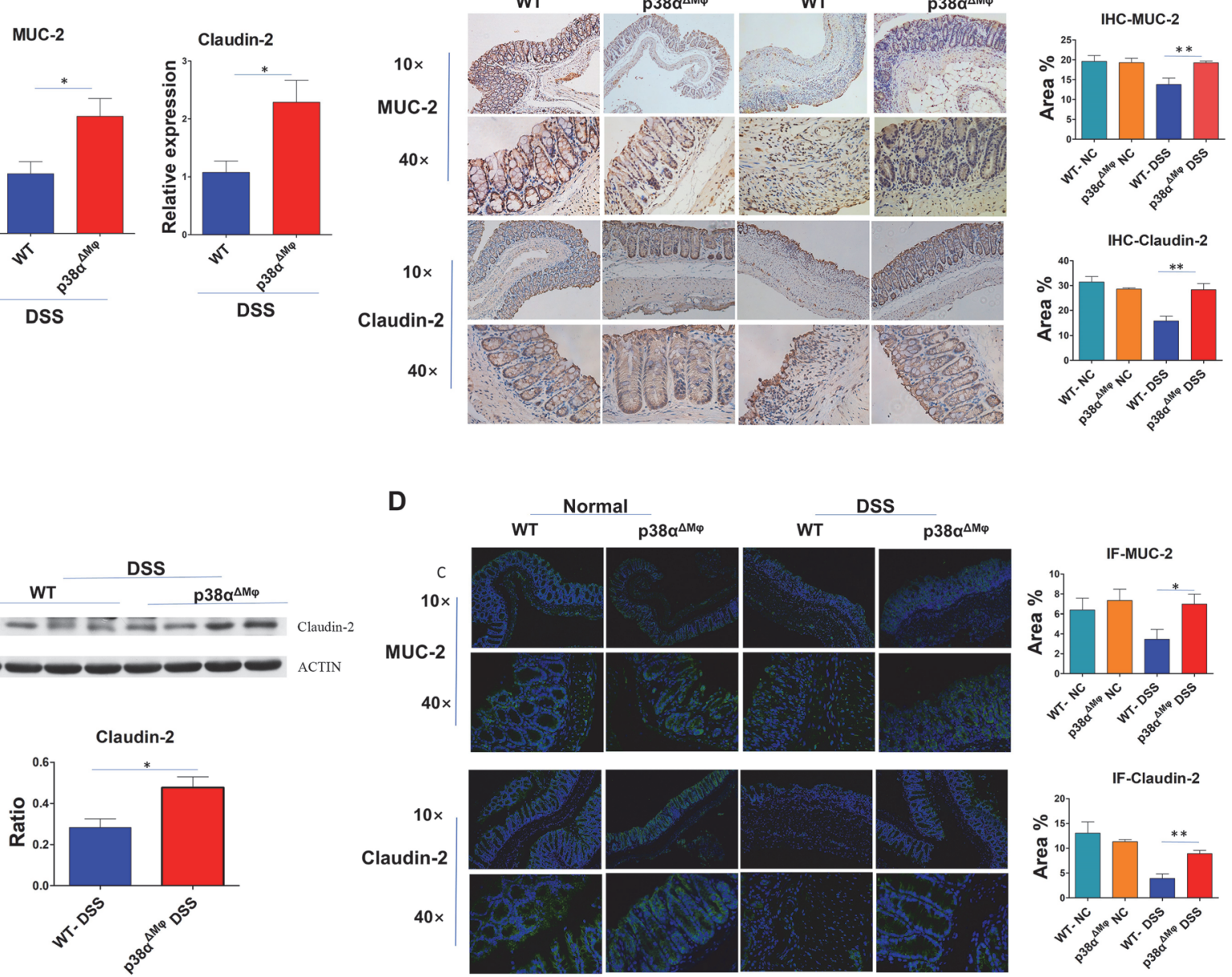

C
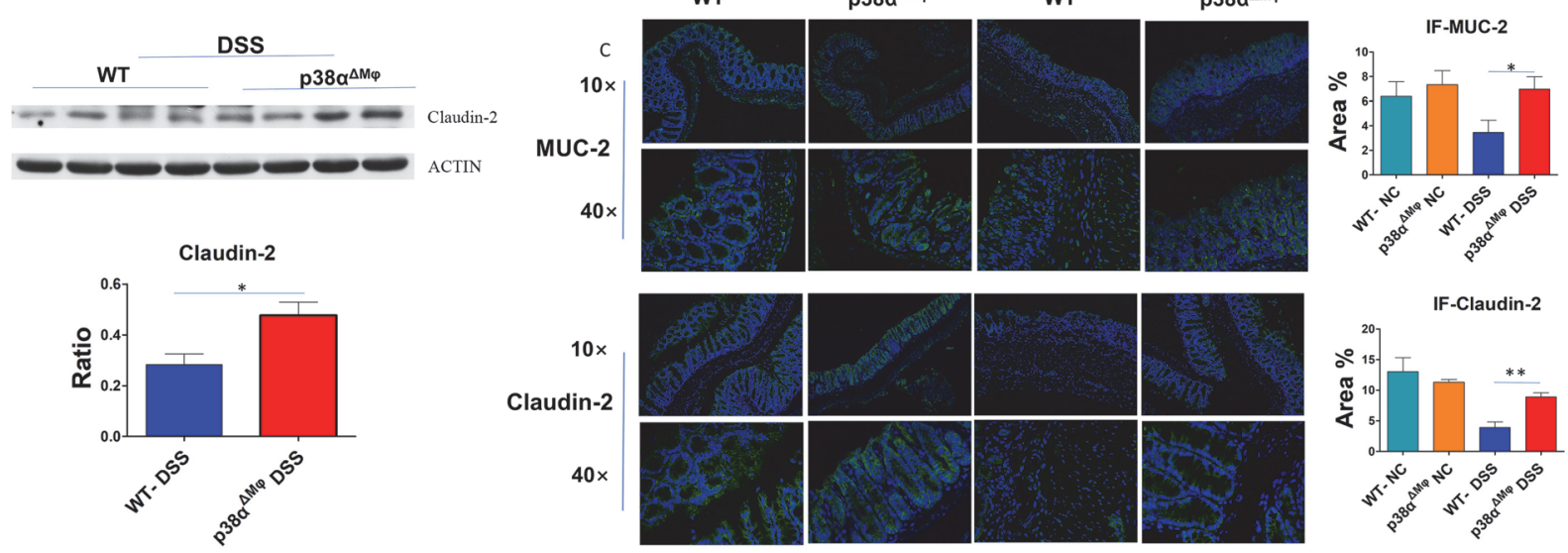

Abstract IDDF2021-ABS-0097 Figure 4

0097 Figure 7. Validation of the immune system processrelated genes between $\mathrm{p} 38 \alpha^{\Delta M \phi}$ and WT groups. (A) The major genes that related to immune system process. (B) The mRNA expression. (C) The protein expression of Oas2 and Oas3. $* P<0.05, * * P<0.01$, and $* * P<0.001)$.

Conclusions Our findings demonstrated that p38 $\alpha$ deficiency in macrophages ameliorated murine experimental colitis by regulating inflammation and the immune process.

A
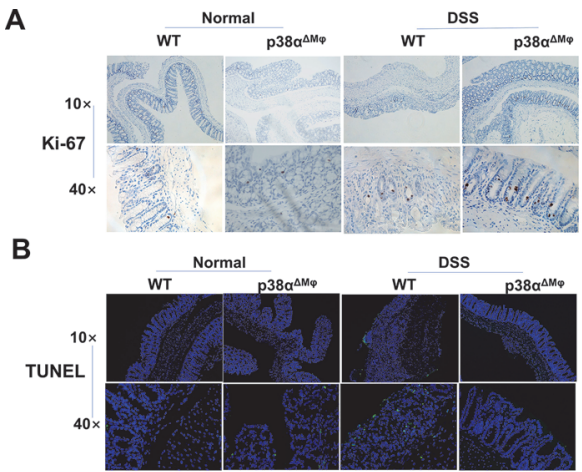

Abstract IDDF2021-ABS-0097 Figure 5
A
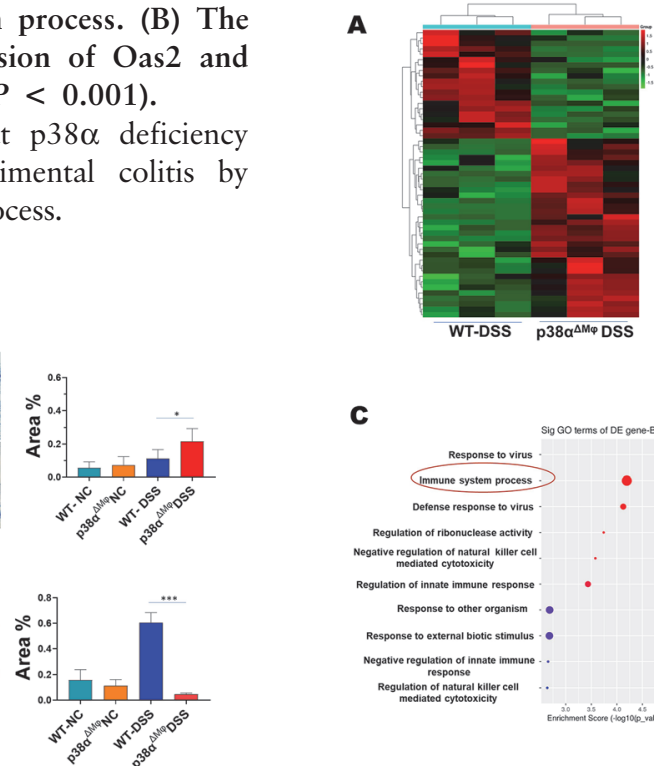

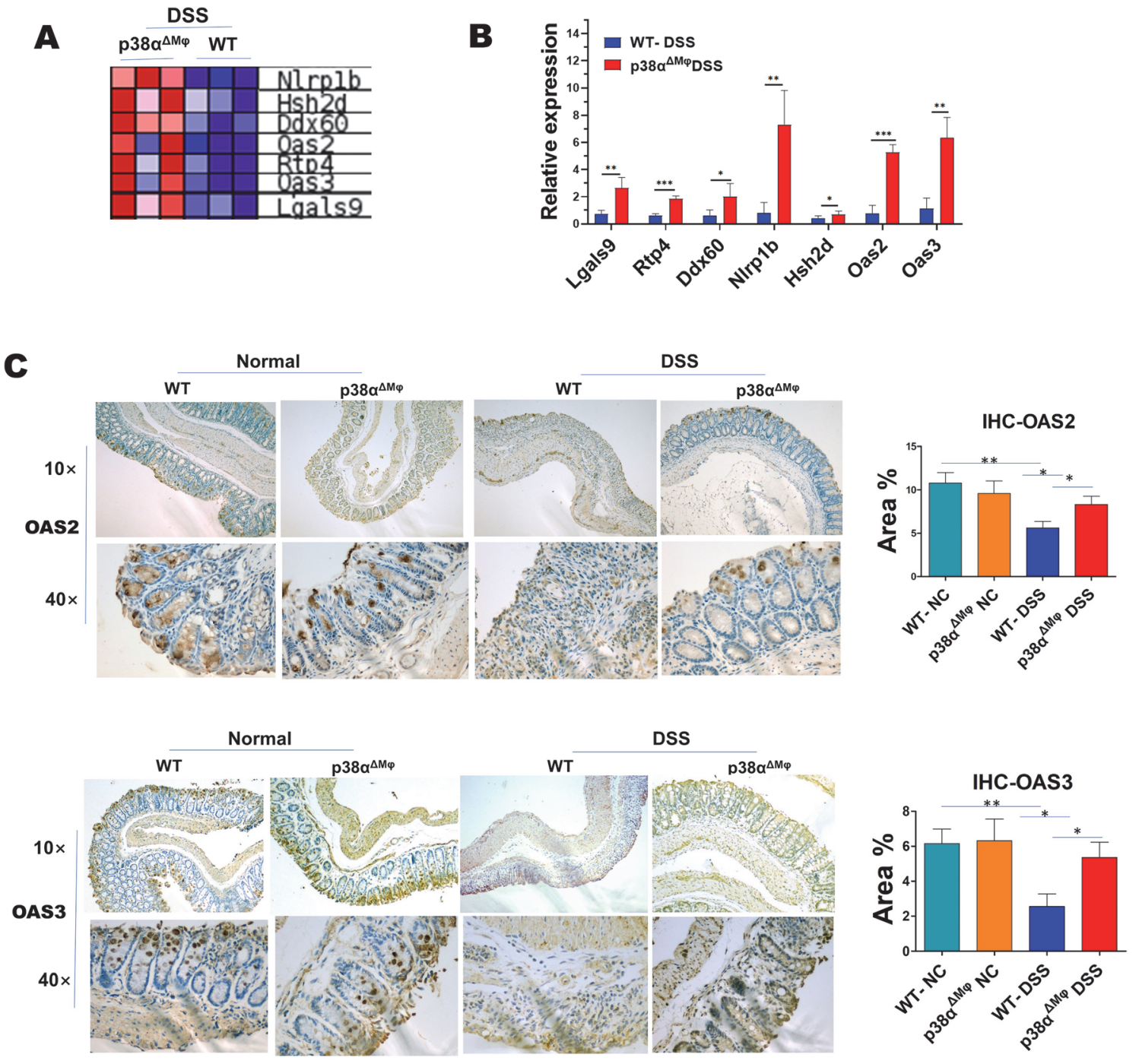

Abstract IDDF2021-ABS-0097 Figure 7

\section{IDDF2021-ABS-0098 COMPARISON OF CURATIVE EFFECT BETWEEN METFORMIN AND MESALAZINE IN THE TREATMENT OF EXPERIMENTAL COLITIS}

${ }^{1}$ Jun Deng* ${ }^{2}$ Lishan Zeng, ${ }^{3}$ Le Liu, ${ }^{3}$ Ye Chen. ${ }^{1}$ The Sixth Affiliated Hospital, Sun Yat-sen University, China; ${ }^{2}$ Guangzhou Panyu Central Hospital, China; ${ }^{3}$ Nanfang Hospital, Southern Medical University, China

\subsection{6/gutjnl-2021-IDDF.35}

Background To evaluate the different efficacy in the treatment of mice colitis between metformin and mesalazine.
Methods C57BL/6 male mice aged 6-8 weeks were given ad libitum access to $3 \%$ dextran sulphate sodium to induce colitis. The treatment groups were administered with metformin and mesalazine, respectively. The body weight, colon length, disease activity index(DAI), histopathological changes and the expression of pro-inflammatory cytokines in each group were evaluated.

Results Compared to the DSS group, mice treated with metformin or mesalazine simultaneously experienced significantly less bodyweight loss, less DAI scores (IDDF2021ABS-0098 Figure 1) and less colon length shortening (IDDF2021-ABS-0098 Figure 2). Histological examination
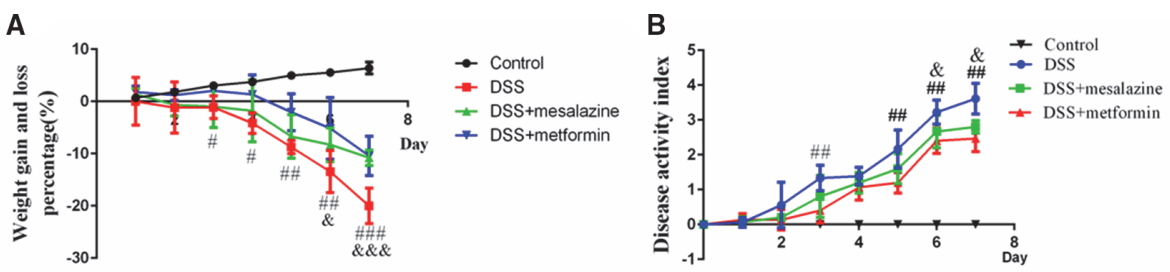